\title{
Dataset overlap density analysis
}

\author{
Andreas H Göller \\ From 8th German Conference on Chemoinformatics: 26 CIC-Workshop \\ Goslar, Germany. 11-13 November 2012
}

The need to compare compound datasets arises from various scenarios, like mergers, library extension programs, gap analysis, combinatorial library design, or estimation of QSAR model applicability domains. Whereas it is relatively easy to find identical compounds in two datasets, the quantification of the overlap is not straightforward. The various approaches described include pairwise nearest neighbor comparisons, clustering and mixed cluster statistics, or binning of e.g. rule-of-five property space distributions. The BCUT methodology creates a binned $\mathrm{N}$-dimensional space and allows to assess the amount of mixed cells. ChemGPS creates a PCA reference projection based on drug-like and satellite molecules in property space to classify new compounds.

But is it possible and also plausible to quantify the overlap of two datasets in a single interpretable number?

PCA projection models with the World Drug Index as drug-like reference space were created based on MACCS, ECFP4, estate or Lipinsky-like physchem descriptors. Compounds from the commercial vendor i-research library, ZINC, ChEMBL and a current screening subset from PubChem were projected onto the WDI maps.

The dataset overlap density index DOD is calculated from the summations over the occupancies of each $\mathrm{N}$-dimensional "volume" element occupied by both datasets, divided by all such elements populated by at least one dataset. The index provides a measure of the overlap of two sets.

It is shown that the number of principal components needed to describe at least $75 \%$ of the information content of the descriptor greatly varies and that a projection in 2 dimensions is not adequate. Such $\mathrm{N}$-dimensional projections are extremely sparse (about 1043 elements for WDI and MACCS descriptor) and crowded only in small regions of the spanned $\mathrm{N}$-dimensional space.

The approach is universal to any descriptor. It can be extended to a DOD vector based on different descriptor

\footnotetext{
Correspondence: andreas.goeller@bayer.com

Bayer Schering Pharma AG, Computational Chemistry, Wuppertal, Germany
}

types each describes different characteristics of the encoded molecules. The box element graining can be easily adjusted as needed for a particular application. based on needs. It allows to quantify local gaps or overlaps. Proprietary datasets can be compared just by the first $\mathrm{N}$ principal component values without even seeing the descriptors behind.

Published: 22 March 2013

doi:10.1186/1758-2946-5-S1-014

Cite this article as: Göller: Dataset overlap density analysis. Journal of

Cheminformatics 2013 5(Suppl 1):014.

\footnotetext{
Publish with ChemistryCentral and every scientist can read your work free of charge

"Open access provides opportunities to our colleagues in other parts of the globe, by allowing anyone to view the content free of charge."

W. Jeffery Hurst, The Hershey Company.

- available free of charge to the entire scientific community

- peer reviewed and published immediately upon acceptance

- cited in PubMed and archived on PubMed Central

- yours - you keep the copyright

Submit your manuscript here:

http://www.chemistrycentral.com/manuscript/

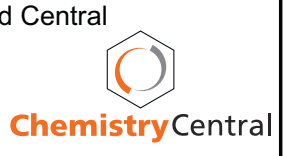

